\title{
Antifibrinolytic Therapy in Ruptured Intracranial Aneurysm Through Repeated Monitoring of Fibrinolytic Activity of Blood
}

\author{
Hiroshi Watanabe, Masanori Ito, Hiroo Chigasaki \\ and Shozo ISHII \\ Department of Neurosurgery, Juntendo University School of Medicine, \\ 3-1-3, Hongo, Bunkyo-ku, Tokyo, Japan
}

\begin{abstract}
Summary
Streptkinase euglobulin lysis time (SK-ELT), fibrin and fibrinogen degradation products (FDP) and fibrinogen in blood were measured to determine the proper dosage of tAMCHA which would not cause ischemic complications but yet would suffice to prevent rebleeding in ruptured intracranial aneurysm. SK-ELT was almost doubled $(130 \mathrm{sec})$ by the administration of $6-8 \mathrm{~g} /$ day of t-AMCHA within $24 \mathrm{~h}$ in normal control. On the other hand, SK-ELT in SAH patients fluctuated markedly during ten days after first bleeding. FDP demonstrated high value soon after first bleeding in Grade IV and V patients. Increase in FDP was also observed following rebleeding, severe vasospasm, and progressive neurological deterioration. Usually the increase in FDP was most obvious between 4-14 days after SAH. Fibrinogen showed the tendency to increase gradually by the administration of t-AMCHA, and this increased fibrinogen would be one of the alarm signs to indicate development of ischemic complications. From these results, it was suggested that doses of t-AMCHA should be determined precisely according to the result of repeated monitoring, and doses should be as such that it could keep SK-ELT constant between $130-150 \mathrm{sec}$.
\end{abstract}

Key words:

ruptured intracranial aneurysm, SAH. fibrinolytic activity, antifibrinolytic therapy, t-AMCHA

\section{Introduction}

It has been well known that there is a high incidence of rebleeding within the first three weeks after a rupture of the intracranial aneurysm. ${ }^{3)}$ It has also been shown that antifibrinolytic agents can decrease the rate of rebleeding. ${ }^{4-8,11)}$ Though tranexamic acid (tAMCHA) has proved to have 10 times as strong an effect as epsilon aminocaproic acid (EACA) in vitro, the doses of this drug necessary to inhibit fibrinolytic activity varies from case to case to a certain degree. The present study was undertaken to investigate a simple method to monitor antifibrinolytic therapy and to determine the proper dosage of t-AMCHA which would not cause ischemic complications but yet would suffice to prevent rebleeding in each individual with ruptured aneurysm.

\section{Materials and Methods}

Thirty-five sequential patients with subarachnoid hemorrhage (SAH) who had been admitted to the department of neurosurgery, Juntendo University School of Medicine, during the period from January through December 1975 were monitored during t-AMCHA therapy; 32 of them had angiographically documented intracranial aneurysms. The period from the last attack to hospitalization and the grade at admission of these 35 patients are shown in Table I. Intravenous t-AMCHA was begun by continuous drip immediately after the lumber puncture had proved existence of SAH. 
Table 1 Interval from the last SAH to admission and the grade on admission of 35 patients with SAH

\begin{tabular}{ccccccc}
$\begin{array}{c}\text { Interval from the last } \\
\begin{array}{c}\text { SAH to admission } \\
\text { (days) }\end{array}\end{array}$ & $\begin{array}{c}\text { Grading at admission } \\
\text { I Hunt \& Hess) }\end{array}$ & II & III & IV & V & Total \\
\hline $0-7$ & 4 & 3 & 8 & 5 & 1 & 21 \\
$8-14$ & 0 & 0 & 1 & 2 & 0 & 3 \\
$15-21$ & 2 & 0 & 0 & 0 & 0 & 2 \\
$22-28$ & 0 & 0 & 0 & 0 & 0 & 0 \\
$29-$ & 9 & 0 & 0 & 0 & 0 & 9
\end{tabular}

Blood sample was obtained from each individual at similar times daily by venipuncture, and citrated plasma was prepared as soon as possible.

SK-ELT: Stock solutions were: 1) cold acetic acid : $0.32 \mathrm{vol}$ of $1 \%$ acetic acid was added to 19 vol. of $\mathrm{H}_{2} \mathrm{O}$ and kept at $4^{\circ} \mathrm{C}$; 2) $1 / 15 \mathrm{M}$ phosphate buffer at $\mathrm{pH} \mathrm{7.4;} \mathrm{3)} 1000$ units of topical thrombin (Mochida Pharmaceutical Co., Ltd) dissolved in $20 \mathrm{ml}$ of $\mathrm{H}_{2} \mathrm{O}$; and 4) 500 units $/ \mathrm{ml}$ streptokinase: 100,000 units of Varidase (Lederle Ltd) was dissolved in $200 \mathrm{ml}$ of $\mathrm{H}_{2} \mathrm{O}$. Euglobulin fraction was prepared as follows: The mixture of $0.5 \mathrm{ml}$ plasma and $9.5 \mathrm{ml}$ cold acetic acid solutin was placed in ice for $30 \mathrm{~min}$. After centrifuging the mixture at 2,000 $\mathrm{g}$ for $10 \mathrm{~min}$, the precipitate was dissolved in $0.5 \mathrm{ml}$ of $1 / 15 \mathrm{M}$ phosphate buffer (euglobulin fraction). SK-ELT was observed by measuring the time ( $\mathrm{sec}$ ) from coagulation to dissolution of the clot which was made by mixing the $0.5 \mathrm{ml}$ euglobulin, $0.1 \mathrm{ml}$ thrombin sol. and $0.1 \mathrm{ml}$ streptokinase sol. at $37^{\circ} \mathrm{C}$.

FDP: Serum FDP was measured by latex coagulation test using Thrombo-Wellcotest (Wellcome Reagents Ltd.)

Fibrinogen: Fibrinogen was measured by thrombin time method using Clotek (Hyland).

\section{Results}

1. Inhibition of the fibrinolytic activity by $t$ AMCHA in control cases

SK-ELT was measured as an indicator of the inhibitory effect of t-AMCHA on the fibrinolytic activity, since the prolongation of SK-ELT has been known to show the decrease of the fibrino- lytic activity. When $8 \mathrm{~g} /$ day of t-AMCHA was continuously administered intravenously to the control patients who had never experienced SAH and who had normal SK-ELT values $(50-60 \mathrm{sec})$, the SK-ELT was prolonged to about twice as long as normal $(120-130 \mathrm{sec})$ within $24-48 \mathrm{~h}$, showing a rapid reduction of fibrinolytic activity (Fig. 1). Only slight prolongation of SK-ELT was shown by the administration of t-AMCHA in the dose of $4 \mathrm{~g}$ /day and it remained less than $100 \mathrm{sec}$ even $48 \mathrm{~h}$ later.

\section{Fluctuation of $S K-E L T$ values in the patients with $S A H$}

Fig. 2 and Fig. 3 show the changes of SK-ELT of the patients in acute stage of SAH (within 7 days) under antifibrinolytic therapy with the changes of patients' grade. SK-ELT of the patients whose neurological grades were I and II (Hunt \& Hess) on admission were prolonged to about twice those of the controls (around $120-130 \mathrm{sec}$ ) within $24-48 \mathrm{~h}$ after intravenous continuous administration of $6-8 \mathrm{~g} / \mathrm{day}$ of $\mathrm{t}$ AMCHA (Fig. 2). After 48 h SK-ELT in most cases were maintained around $130-150 \mathrm{sec}$ by the administration of the same doses of tAMCHA. This fact indicates that the reduction of fibrinolytic activity in Grade I or II patients is easily achieved by the use of t-AMCHA (6-8 g/day) for 1-2 days, and also it is not so difficult to keep the reduced fibrinolytic activity fairly stable thereafter. Even in such cases, however as shown in one case (Fig. 2), when vaso-

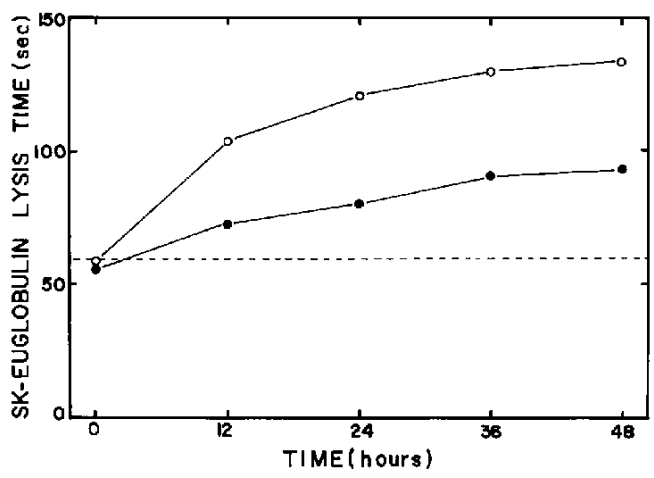

Fig. 1 Prolongation of SK-euglobulin lysis time by the use of t-AMCHA in control cases. t-AMCHA was administered intravenously by continuous drip infusion. Open circle $O: 8 \mathrm{~g} /$ day of $\mathbf{t}-$ AMCHA, closed circle $\mathrm{O}$ : $\mathrm{g} / \mathrm{day}$ of $\mathrm{t}-\mathrm{AMCHA}$ 


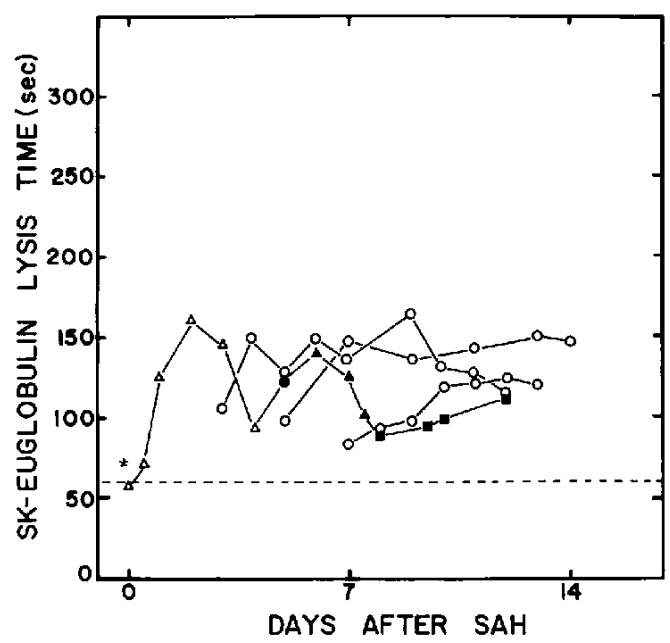

Fig. 2 Changes of SK-ELT value in the patients with SAH. Changes of SK-ELT in the patients whose neurological grades were I and II on admission were summarized with the changes of patients' grade. $\bigcirc:$ grade I, $\triangle:$ grade II, $\bigcirc$ : grade IIl, : grade IV, $\square$ : grade $V$

spasm appeared on the 4th day after SAH, SKELT showed rapid reduction after having once achieved the value of $150 \mathrm{sec}$ followed by marked fluctuation in association with the deterioration of neurological signs. This fact suggests that vasospasm or some factors associated with vasospasm might be responsible for the activation of the fibrinolytic system. Fig. 3 shows the changes of SK-ELT in patients who were admit-

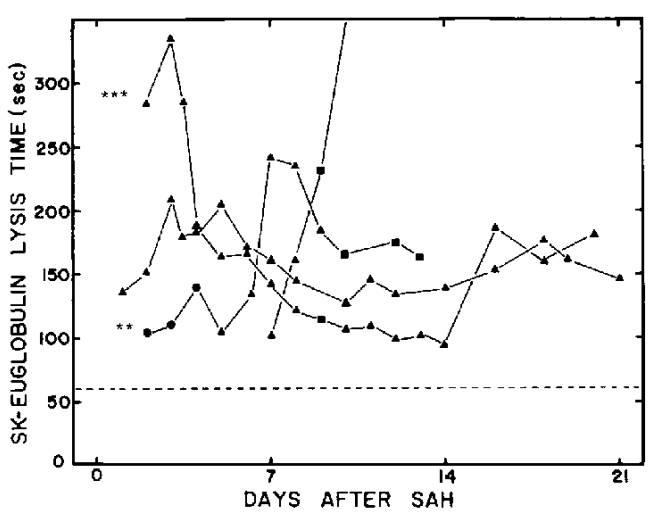

Fig. 3 Changes of SK-ELT value in the patients with SAH. Changes of SK-ELT in the patients whose neurological grade were III to $\mathrm{V}$ at admission were summarized with the changes of patients' grade. $\bigcirc$ : grade $I, \triangle$ : grade $I 1, \bigcirc$ : grade III. : grade IV, $\mathbf{\square}$ : grade $\mathrm{V}$ ted within one week of $\mathrm{SAH}$ in condition of Grade III or IV. Some patients showed low SKELT (around $100 \mathrm{sec}$ ) even after the administration of t-AMCHA for several days (Fig. 3) and others showed values of more than $250 \mathrm{sec}$ even in the first day of admission (Fig. 3). Remarkable fluctuation of the SK-ELT was observed until 10th day of SAH in advanced grade patients with as compared with those of Grades I or II, and a positive correlation between SKELT values and dosages of t-AMCHA was not always noticeable. These suggest that many factors may influence the SK-ELT value in acute stage of SAH, especially in advanced grade patients.

No marked fluctuation of SK-ELT was observed in patients admitted later than two weeks after SAH (data not shown in Figure).

\section{Increased FDP as an alarming sign for raised fibrinolysis}

Fig. 4 shows the values of blood FDP with changes of patients' grade in acute stage of $\mathrm{SAH}$. FDP in most patients who showed mild neurological symptoms (Grade I-III) when SAH did occur, was found to be less than $10 \mu \mathrm{g} / \mathrm{ml}$ early in stage, and thereafter it remained within normal range (less than $7 \mu \mathrm{g} / \mathrm{ml}$ ) as long as they took comparatively favorable courses.

On the other hand, most Grade IV and V patients at the time of SAH showed high FDP values $(10-30 \mu \mathrm{g} / \mathrm{ml})$ from the early stage. This high FDP value observed in the early stage returned to normal in a few days in most cases unless neurological conditions deteriorated. FDP which had returned once to the normal range, however, increased again around one week in certain instances, as shown in Fig. 4. One of them (Fig. 3,4) is the case in which rebleeding from the aneurysm occurred on the 4th day, and the other (Fig. 3,4) is the case in which continuous minor leakage from the aneurysm was suspected because of the persisting bloody CSF.

In either case FDP took high value from the first day of the attack. Once returning to the normal level, their FDP rose again on the $4 t h$ day, then fluctuated considerably keeping high values for following two weeks. FDP also rose in association with the occurrence of vasospasm. One representative case was shown in Fig. 4. In 


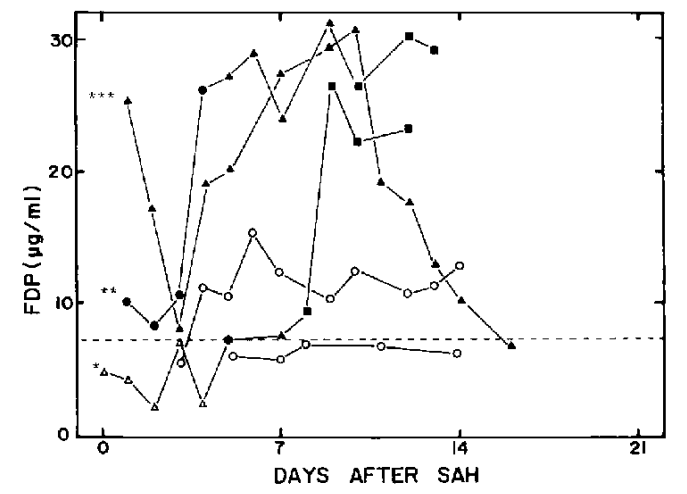

Fig. 4 Fluctuation of blood FDP in the patients with SAH. Changes of FDP in acute stage of SAH were summarized with the changes of patients' grade. $\bigcirc:$ grade I, $\triangle$ : grade II, $\bigcirc$ : grade III, : grade $\mathrm{IV}, \boldsymbol{\square}$ : grade $\mathrm{V}$

this case (rt-ICPC aneurysm) the patient was admitted on the day of SAH in Grade II and no vasospasm was detected in four vessel angiography. Though FDP remained at normal value, consciousness disturbance began from the 5 th day and mild vasospasm was demonstrated angiographically on the 6th day. Thereafter the neurological deterioration and vasospasm progressed rapidly and marked FDP increase was observed from the 8th day. The fact that the increase in FDP was almost exclusively found between 4-14 days after SAH is very interesting because this period correlates well with the time when the rebleeding of the aneurysm or vasospasm develops most frequently.

\section{Decrease of fibrinogen following the acti- vation of fibrinolytic activity}

Positive correlation was not observed between the grade of patients and the fibrinogen contents measured on admission in patients who were hospitalized within 7 days of SAH. Fibrinogen values of these patients ranged from 180 to $540 \mathrm{mg} / \mathrm{dl}$ with the mean of $338 \mathrm{mg} / \mathrm{dl}$. Though marked fluctuation was rarely observed in the level of fibrinogen even in acute stage of $\mathrm{SAH}$, fibrinogen showed the tendency to increase gradually by the use of antifibrinolytic agent (t-AMCHA).

On the other hand, reduction of fibrinogen was observed in association with marked vasospasm and accompanied neurological deterioration. The decrease of fibrinogen was also found in the case of rebleeding. Decreased value of fibrinogen reached $60-120 \mathrm{mg} / \mathrm{dl}$, and this decrease was always accompanied by the increase of FDP. These facts may indicate that observed decrease of fibrinogen is probably due to the increased fibrinolytic activity in association with vasospasm or rebleeding.

\section{Protective effect of $t-A M C H A$ against re- bleeding}

Only one of 32 sequential aneurysm patients treated with $\mathrm{t}$-AMCHA rebled during the period of monitoring. This patient had a second attack 4 days after the first episode when the value of SK-ELT was $104 \mathrm{sec}$ in spite of t-AMCHA use in dosages of $8 \mathrm{~g}$ /day for 3 days. Two factors might be responsible for the rebleeding: one is that the amount of t-AMCHA administered was not enough to suppress the fibrinolytic activity because of the unusual activation of plasmin. The other is the use of hypotonic dehydrator. Since urinary excretion of t-AMCHA is acceralated due to diuresis, one must consider the possibility that the concentration of $\mathrm{t}-\mathrm{AMCHA}$ in blood does not increase in proportion to the dose of t-AMCHA. The rate of rebleeding, $3.1 \%$, in our series is very low in comparison with others when considering the fact that 22 of 32 patients were admitted within 2 weeks of SAH.

\section{Discussion}

"How to prevent the rebleeding" is one of the most fundamental issues in the management of ruptured aneurysm. Rebleeding has been observed in $6-22 \%$ ) even during two weeks waiting period for surgery. This high rate of rebleeding gives the important basis for an early operation. Recently there has been some reports mentioning favorable surgical results when it is performed within $48 \mathrm{~h}$ after the first attack. ${ }^{91}$ However, the results of early operation performed within one week is distinctly less satisfactory when compared to operation conducted after two weeks. ${ }^{2)}$ Thus, this fact provides the main basis for delayed surgery. Particularly in cases of the progressive vasospasm associated with the neurological deterioration, we are often obliged to delay surgery with attempts to prevent rebleeding and to improve the general conditions at the same time. 
Concerning antifibrinolytic therapy, there has been many reports of its effectiveness in preventing rebleeding from the ruptured intracranial aneurysm. ${ }^{4}$ 8.10) Many problems, however, remain unsettled, such as determination of sufficient antifibrinolytic agent dosage to prevent rebleeding and still to avoid such side effects as ischemia or infarction, and also to prevent rebound fibrinolysis etc. Therefore, it would be important to monitor each case within some parameters which can correctly evaluate the effect of the antifibrinolytic agents.

Though monitoring the effect of antifibrinolytic therapy has been reported by some authors, ${ }^{1.5 .8)}$ these methods have some points that require improvement: some of them are too time consuming and the others are not adequate in finding out the anticipated decrease of fibrinolytic activity due to antifibrinolytic agents. On the other hand, the SK-ELT measurement has various advantages; eg. the euglobulin fraction prepared from plasma by isoelectric sedimentation at $\mathrm{pH} 5.2$ does not contain the naturally cxisting inhibitors in the fibrinolytic system, and the reaction has been accelerated through the activation of proactivators by adding streptokinase to shorten measurement time. By this method, the measurement can be finished within 60 min from blood withdrawal.

Monitoring the effect of antifibrinolytic therapy revealed that there was marked fluctuation in both SK-ELT and FDP in the acute stage of SAH (4-14 days after SAH, especically in advanced grade patients. Clinically, this period coincides with the time of rebleeding and with that of appearance of ishemic complications due to vasospasm. Thus repeated monitoring and consequent alterations of 1-AMCHA dosage would be necessary in managing acute stages of ruptured aneurysm.

The value of SK-ELT expresses indirectly the effect of t-AMCHA upon the fibrinolytic activity. In those cases whose SK-ELT remains around $100 \mathrm{sec}$ for several days, in spite of the antifibrinolytic therapy, the possibility of rebleeding is high and prescribing an additional amount of t-AMCHA should be considered. As described before, the use of a large amount of hypertonic dehydrator would mostly be responsible for this low SK-ELT. Therefore, from the standpoint of antifibrinolytic therapy, external drainage or shunt operation would be more recommendable than the use of hypertonic dehydrator in the control of increased ICP.

On the other hand, at least factors should be kept in mind for abnormal prolongation of SKELT; The first is an overdose of t-AMCHA. In one patient from the control group, SK-ELT increased to $250 \mathrm{sec}$ within 3 days by the administration of t-AMCHA in dosages of $12 \mathrm{~g}$ /days. The second is a decrease of urine output. In those cases whose urine volume is less than $800 \mathrm{ml} /$ day (in adult,) the accumulation of $\mathrm{t}$ AMCHA would occur in spite of the use of relatively smaller doses of the agent. The third is increased fibrinogen in blood. Two reasons could be considered for this high fibrinogen. One is hemoconcentration due to dehydration which is frequently observed in patients with severe disturbance in consciousness. The other is the one induced by the use of t-AMCHA. Although the reason for the increase in fibrinogen following t-AMCHA therapy has not as yet been fully explained, the decrease in fibrinolysis is the most reasonable explanation.

At any event, the accumulation of $\mathrm{t}-\mathrm{AMCHA}$ in blood would induce an increase in blood viscosity through the fibrinogen increase, and it would be responsible for the decrease of microcirculation in the brain. Since a marked prolongation of SK-ELT is mostly seen in poor grade patients, the determination of the minimum necessary dosage of t-AMCHA for these patients with such disturbed microcirculation seems to be very important.

Details of changes of fibrinogen in blood and of blood viscosity during antifibrinolytic therapy will be reported later. From our experiences, it becomes clear that the amount of t-AMCHA should be as such that SK-ELT can be kept between $130-150 \mathrm{sec}$ in the acute stage of SAH.

Since the time of increase in FDP observed in this study well correlates with the time of frequent occurrence of rebleeding or vasospasm, this must be considered as an important alarm sign.

\section{References}

1) Geronemus, R., Herz, D. A. and Shulman, K.: Streptokinase clot lysis time in patients with ruptured intracranial aneurysms. J. Neurosurg. 40: 499-503, 1974

2) Krayenbühl, H. A., Yasargil, M. G., Flamm, E. S. and Tew, J. M.: Microsurgical treatment of 
intracranial saccular aneurysm, $J$. Neurosurg. 37: 678-686, 1972

3) Locksley, H. B.: Natural history of subarachnoid hemorrhage, intracranial aneurysm and arteriovenous malformation, $J$. Neurosurg. 25: $321-368,1966$

4) Mullan, S. and Dawley, J.: Antifibrinolytic therapy for intracranial aneurysm, J. Neurosurg. 28 : $21-23,1968$

5) Nibbelink, D. W.: Antifibrinolytic activity during administration of epsilon-aminocaproic acid, Stroke 2: 555-558, 1971

6) Norlén, G. and Thulin, C. A.: The use of antifibrinolytic substances in ruptured intracranial aneurysms, Neurochirurgia (Stuttgart)
12: 100-102, 1969

7) Ransohoff, J. M., Goodgold, A. and Benjamin, M. V.: Preoperative management of patients with ruptured intracranial aneurysms, J. Neurosurg. 36: 525-530, 1972

8) Smith, R. R. and Upchurch, J. J.: Monitoring antifibrinolytic therapy in subarachnoid hemorthage, J. Neurosurg. 38: 339-344, 1973

9) Suzuki, J. and Yoshimoto, T.: Early operation for the ruptured intracranial aneurysms, Neurological Surgery 4: 135-141, 1976

10) Tovi, D.: The use of antifibrinolytic drugs to prevent early recurrent aneurysmal subarachnoid hemorrhage, Acta neurol. scand. 49: 163-175, 1973 